\title{
EDUCA cuevas y EDUCA centros: programa educativo de la red de Cuevas Prehistóricas y Centros Culturales de Cantabria
}

\begin{abstract}
Recientemente se ha presentado el proyecto EDUCA cuevas y EDUCA centros, un recurso didáctico del área de difusión de la red de Cuevas Prehistóricas y Centros de Interpretación de Cantabria, destinado al alumnado y profesorado de los centros educativos en las etapas de educación primaria y educación secundaria obligatoria. Esta propuesta didáctica desde el ámbito de la educación patrimonial persigue el conocimiento y la comprensión del patrimonio cultural por medio de la participación activa de las personas con necesidades educativas.
\end{abstract}

Alberto Peña Fernández | Cuevas Prehistóricas y Centros Culturales de Cantabria. S.RECD

URL de la contribución <www.iaph.es/revistaph/index.php/revistaph/article/view/3555>

La red de cuevas prehistóricas con arte rupestre declaradas patrimonio mundial por la UNESCO en julio de $2008^{1} \mathrm{y}$ los centros de interpretación, conjunto de infraestructuras culturales de titularidad autonómica y gestionados por la Consejería de Educación, Cultura y Deporte, a través de la Sociedad Regional de Educación, Cultura y Deporte de Cantabria (S.RECD), constituyen una atractiva oferta de turismo cultural sustentada en la puesta en valor de estos enclaves para permitir su accesibilidad y comprensión.

Dejando al margen la investigación y conservación de estos bienes culturales, como dos de los activos imprescindibles en la gestión del patrimonio, nos centraremos aquí en la difusión con fines didácticos y educativos de estos yacimientos y centros de interpretación, en especial, dando a conocer a la sociedad en general y a los museólogos, gestores culturales, educadores, docentes, etc., en particular, la nueva programación educativa que complementa las visitas guiadas, teatralizadas en algún caso y talleres didácticos que vienen ofertándose tradicionalmente en estos espacios patrimoniales.

Este programa educativo se enriquece desde este año con la puesta en marcha de EDUCA cuevas y EDUCA centros, espacio dedicado al conocimiento de estos sitios de patrimonio por medio de una serie de herramientas y estratégicas en el ámbito de la educación no formal, que pretenden acercar la historia y el patrimonio cultural de la comunidad autónoma a los centros educativos y a las personas con necesidades de aprendizaje ${ }^{2}$.
Se trata de una serie de cuadernos didácticos del alumno y del profesorado que ya están disponibles en las dos plataformas virtuales de cuevas y centros culturales ${ }^{3}$ y que desarrollan una estructura uniforme de contenidos, comenzando con una introducción sobre el yacimiento o centro, con un personaje animado y representativo del sitio para la etapa de educación primaria, objetivos curriculares de etapa y competencias, actividades que combinan lo educativo con lo lúdico, ejercicios de comprensión y pasatiempos diversos. En lo que respecta al cuaderno del profesor, tras la presentación del marco geográfico y crono cultural del yacimiento o centro, continúa un apartado de orientaciones didácticas sobre el aprovechamiento del recurso, recomendaciones de uso, información práctica para desarrollar itinerarios, descripción del bien en sus diferentes coordenadas espacio temporales y finalmente la bibliografía básica para completar la actividad en el aula y en la visita.

Esta oferta educativa se articula en una serie de itinerarios que permiten al alumnado, atendiendo al diseño curricular y a las diferentes etapas no universitarias, acercarse desde la observación, interpretación, investigación teórica y experimentación práctica a los diferentes testimonios materiales e inmateriales del patrimonio cultural de Cantabria, mediante una metodología diferente, pero complementaria a la empleada en la educación formal. Estos itinerarios, que se corresponden con los diferentes periodos históricos y ejes temáticos (arqueología, arte e historia) son los siguientes: 
$>$ Vive la Prehistoria: cuevas con arte rupestre paleolítico. $>$ Entramos en la historia. Cántabros y romanos: Domus de Julióbriga y Arqueositio de Camesa-Rebolledo.

$>$ Cantabria medieval: centro de interpretación del románico de Villacantid y torre de Pero Niño.

La metodología se basa en el desarrollo de tres fases interrelacionadas. Una primera fase de preparación en el aula del lugar que van a visitar y algunos de los contenidos que serán abordados en la visita; una segunda fase de escucha y participación activa de los estudiantes durante la visita al yacimiento o centro de interpretación, donde el guía comunicará los contenidos y claves interpretativas necesarias para, ya en una tercera fase, después de la visita, ser capaz de plasmar lo aprendido y experimentado en la visita y en el taller, en los cuadernos didácticos.

Laescasa presencia en los planes docentes de contenidos relacionados con la historia y el patrimonio de Cantabria, según se desprende del informe del Observatorio del Patrimonio en Cantabria, refuerza esta propuesta didáctica, que complementa a las visitas y talleres ofertados hasta ahora como estrategias de difusión ${ }^{4}$. Esta primera etapa, pendiente de evaluar y revisar periódicamente, ha supuesto la documentación, diseño e implementación de un total de veinticuatro unidades didácticas, quedando pendiente para una segunda fase los cuadernos del alumnado y profesorado de la etapa de bachillerato que se desarrollará a lo largo del curso 2014-15. Este proyecto ha sido coordinado por Daniel Garrido Pimentel, coordinador de la red de Cuevas Prehistóricas y Centros Culturales de Cantabria; desarrollado por Alberto Peña Fernández, guía cultural; y revisado en sus aspectos pedagógicos y metodológicos por Miguel A. García de la Santa y Mikel Echeita Blanco, de la Dirección General de Ordenación e Innovación Educativa de la Consejería de Educación del Gobierno de Cantabria. Además ha contado con la colaboración altruista de ilustradores y dibujantes, asesoramiento de historiadores, arqueólogos, docentes y gestores culturales.
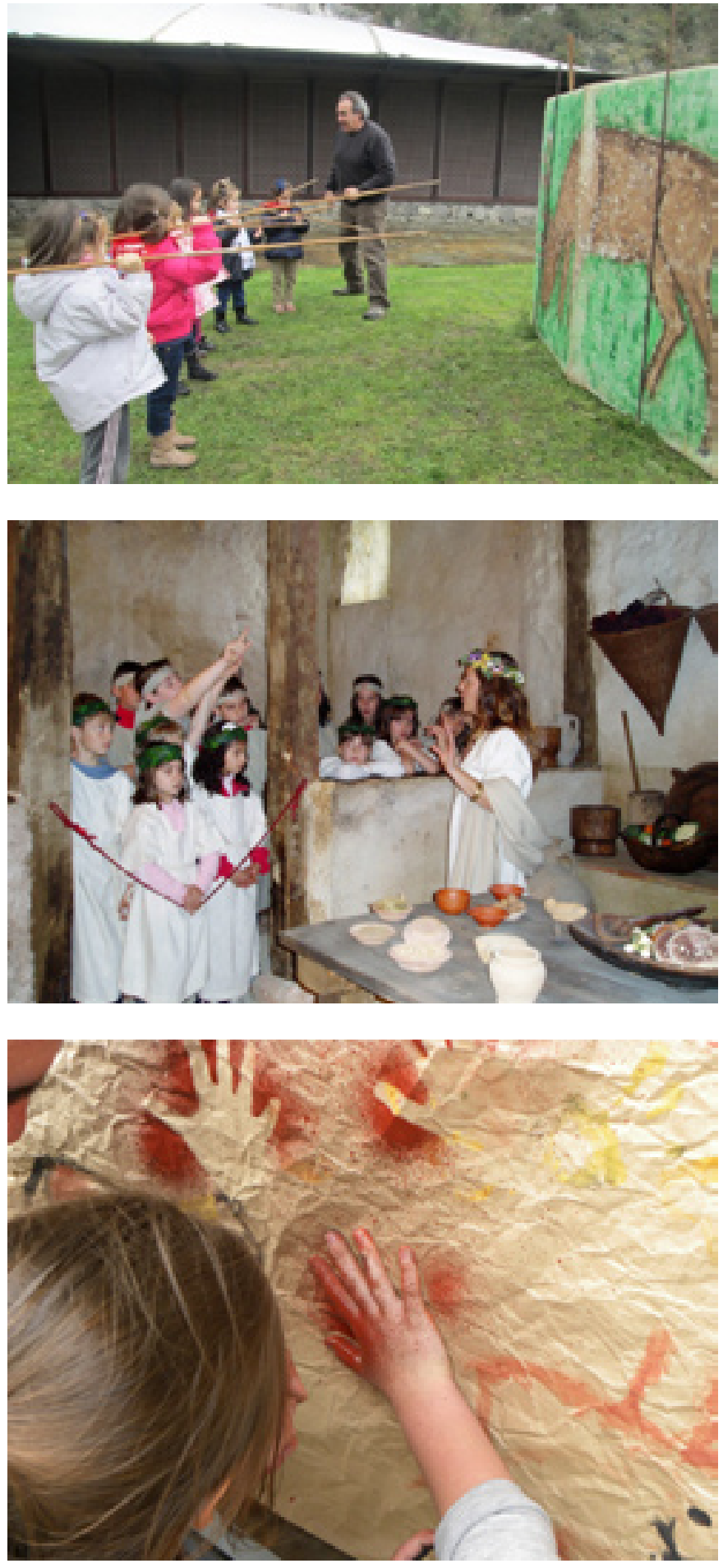

Taller Los cazadores de las cuevas pintadas (superior), visita teatralizada a la domus romana de Julióbriga (central) y taller El primer grafiti (inferior) | fotos Cuevas Prehistóricas y Centros Culturales de Cantabria 

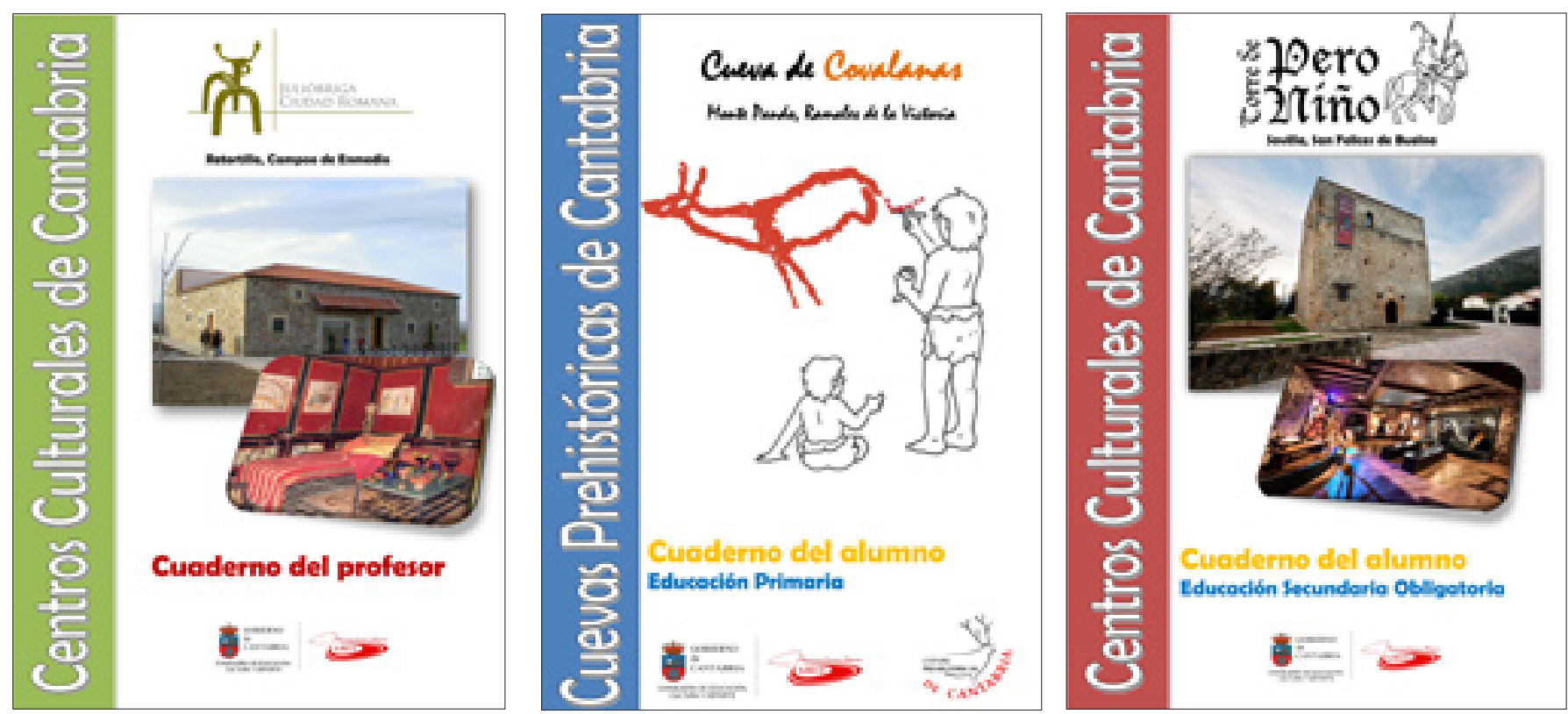

Cuadernos didácticos del programa EDUCA Cuevas y EDUCA Centros

\section{NOTAS}

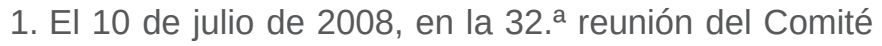
del Patrimonio Mundial de la Organización de las Naciones Unidas para la Educación, la Ciencia y la Cultura (UNESCO) celebrada en Quebec, nueve cuevas prehistóricas de Cantabria fueron declaradas patrimonio mundial, dentro del sitio Cueva de Altamira y arte rupestre paleolítico del Norte de España.

2. El programa incluye las cuevas con arte rupestre del Castillo, las Monedas, Covalanas y el Pendo, así como los centros de interpretación Domus romana de Julióbriga, Arqueositio de Camesa-Rebolledo, centro de interpretación del Románico de Villacantid y torre de Pero Niño.

3. Cuevas Prehistóricas de Cantabria <http://cuevas. culturadecantabria.com/ $>$ y Centros Culturales de Cantabria <http://centros.culturadecantabria.com>.

4. El Observatorio del Patrimonio en Cantabria es un programa puesto en marcha por el Aula de Patrimonio Cultural de la Universidad de Cantabria en 2013. Una de sus principales conclusiones, tras una fase previa de diagnóstico, es que los planes docentes actuales no contemplan horas para contenidos sobre el patrimonio cultural y se carece de optativas para que los alumnos más interesados puedan perfilar su formación hacia ese campo de conocimiento. 